\title{
Aquaplanets, Climate Sensitivity, and Low Clouds
}

\author{
Brian Medeiros and Bjorn Stevens \\ University of California, Los Angeles, Los Angeles, California \\ IsAaC M. Held AND Ming ZhaO \\ Geophysical Fluid Dynamics Laboratory, Princeton, New Jersey \\ DAVID L. Williamson AND JERRY G. OLSON \\ National Center for Atmospheric Research, Boulder, Colorado \\ CHRistopher S. BRETHERTON \\ University of Washington, Seattle, Washington
}

(Manuscript received 17 April 2007, in final form 6 March 2008)

\begin{abstract}
Cloud effects have repeatedly been pointed out as the leading source of uncertainty in projections of future climate, yet clouds remain poorly understood and simulated in climate models. Aquaplanets provide a simplified framework for comparing and understanding cloud effects, and how they are partitioned as a function of regime, in large-scale models. This work uses two climate models to demonstrate that aquaplanets can successfully predict a climate model's sensitivity to an idealized climate change. For both models, aquaplanet climate sensitivity is similar to that of the realistic configuration. Tropical low clouds appear to play a leading role in determining the sensitivity. Regions of large-scale subsidence, which cover much of the tropics, are most directly responsible for the differences between the models. Although cloud effects and climate sensitivity are similar for aquaplanets and realistic configurations, the aquaplanets lack persistent stratocumulus in the tropical atmosphere. This, and an additional analysis of the cloud response in the realistically configured simulations, suggests the representation of shallow (trade wind) cumulus convection, which is ubiquitous in the tropics, is largely responsible for differences in the simulated climate sensitivity of these two models.
\end{abstract}

\section{Introduction}

Clouds alter the sensitivity of the climate system to external perturbations. Understanding and quantifying how they do so has been identified as imperative for several decades, yet cloud effects remain the largest contribution to uncertainty in climate sensitivity (Arakawa 1975; Charney et al. 1979; Houghton et al. 2001; Randall et al. 2007). Notwithstanding the ever-increasing complexity of general circulation models (GCMs)

Corresponding author address: Brian Medeiros, Department of Atmospheric and Oceanic Sciences, University of California, Los Angeles, Box 951565, 405 Hilgard Ave., Los Angeles, CA 900951565.

E-mail: brianpm@ucla.edu and the steady progression in our understanding of cloud-scale processes, insights into how clouds modulate climate sensitivity have been few. Those insights that have developed often are expressed in the form of simple models of the tropical atmosphere (e.g., Miller 1997; Larson et al. 1999); determining the relevance of these models to simulations with full physics GCMs is nontrivial. This disparity provokes the question as to whether an intermediate framework-the aquaplanet-might present an opportunity to better understand cloud effects on the climate sensitivity of GCMs, and in so doing help bridge this gap.

Aquaplanets are similar to Earth, except with a uniformly water-covered surface. They have been used in a number of contexts in atmospheric modeling studies (e.g., Hayashi and Sumi 1986; Kirtman and Schneider 
2000; Chao and Chen 2004; Miura et al. 2005). The aquaplanets used here are forced by a family of prescribed sea surface temperature (SST) distributions that vary only with latitude. There is no sea ice, and perpetual equinoctial orbital parameters remove seasonal variations in insolation; diurnal variability is preserved. Although the boundary conditions are simplified, the full GCM dynamics and physics are retained.

To what extent can a GCM with aquaplanet boundary conditions be used to predict the climate sensitivity of that same GCM forced with more Earth-like boundary conditions? If aquaplanets are robust predictors of a GCM's climate sensitivity, one can hope to exploit their relative simplicity (e.g., short equilibration times, hemispheric and zonal symmetry that reduce the climate's dimensionality) to develop a deeper understanding of how clouds affect the climate sensitivity of GCMs.

Irrespective of their correspondence to more Earthlike simulations, aquaplanets are useful for exploring the relative role of zonally symmetric, versus asymmetric, circulations. Among the tropical boundary layer cloud regimes, shallow (or trade wind) cumulus cover large expanses of the trades and could be associated with the zonally symmetric component of the overturning circulation. Stratocumulus, however, tend to be confined to eastern boundary current regions where the effect of the neighboring landmass tends to favor cooler surface waters and warmer free-tropospheric temperatures. As such, stratocumulus are more characteristic of the zonally asymmetric component of the overturning circulation. Through their zonal symmetry, one thus expects aquaplanets to emphasize trade wind cumulus at the expense of stratocumulus, thereby helping delineate the relative role of these cloud types in determining the overall tropical cloud response.

In this work, several aquaplanet configurations are used in two independently developed GCMs to determine if the framework is a suitable predictor of climate sensitivity. Section 2 introduces the models and details the experimental design. Section 3 explores the climate of aquaplanets and their response to an increase in SST. Following that, section 4 discusses climate sensitivity and cloud response, where the climate sensitivity of aquaplanet configurations is shown to be similar to realistically configured GCMs. Section 5 compares the cloud response of the GCMs, showing that changes in low-cloud amount are consistent with tropically averaged changes in cloud radiative forcing, and that regions of large-scale subsidence are most important for these cloud effects. Section 6 extends the ideas of the previous sections, connecting the thermodynamic and dynamic effects on changes in cloud radiative forcing to the response in particular large-scale regimes. This analysis suggests that shallow cumulus convection is the most relevant cloud type in the aquaplanet response, and likely it is at least as important for realistic simulations as other low-cloud types, including stratocumulus. Section 7 provides a brief summary.

\section{Methods}

The two GCMs used in this study are the National Center for Atmospheric Research (NCAR) Community Atmosphere Model (CAM) and the Geophysical Fluid Dynamics Laboratory (GFDL) Atmosphere Model (AM), the atmospheric components of fully coupled climate models [Community Climate System Model (CCSM) and global coupled climate model (CM), respectively]. These models have been compared in other contexts, as have previously released versions, and there are well-recognized differences between their simulated climates, both in the mean state and in climate variability (e.g., Wyant et al. 2006). Cloud effects in these GCMs are dramatically different, as discussed herein and in previous studies; the different cloud effects are representative of the disagreement among many current GCMs (cf., Soden and Held 2006). Full descriptions of these atmospheric GCMs can be found elsewhere (Collins et al. 2006; Delworth et al. 2006), but it is interesting to point out some relevant differences. The numerical methods used to solve the equations of motion are fundamentally different, with the NCAR CAM being a spectral model and the GFDL AM being a gridpoint model. Both GCMs employ a mass flux scheme for deep, moist convection, but in somewhat different implementations. The NCAR CAM uses a Zhang-MacFarlane scheme (Zhang and MacFarlane 1995) for deep convection [and a separate three-level scheme for shallow convection, following Hack (1994)] and the GFDL AM uses a relaxed Arakawa-Schubert scheme based on Moorthi and Suarez (1992). The treatment of boundary layer clouds is important for climate sensitivity, and the GCMs differ substantially in this regard. Cloud liquid and ice are determined prognostically by both models, based on different microphysical schemes. The boundary layer schemes differ, with the NCAR CAM applying the nonlocal scheme of Holtslag and Boville (1993), while the GFDL AM uses a Kprofile scheme motivated by large-eddy simulations (Lock et al. 2000) coupled to a local mixing scheme for stable layers. The determination of cloud fraction is also quite different; the NCAR CAM diagnoses cloud fraction from convective mass flux, relative humidity, and large-scale stability, while the GFDL AM employs the prognostic scheme of Tiedtke (1993). 


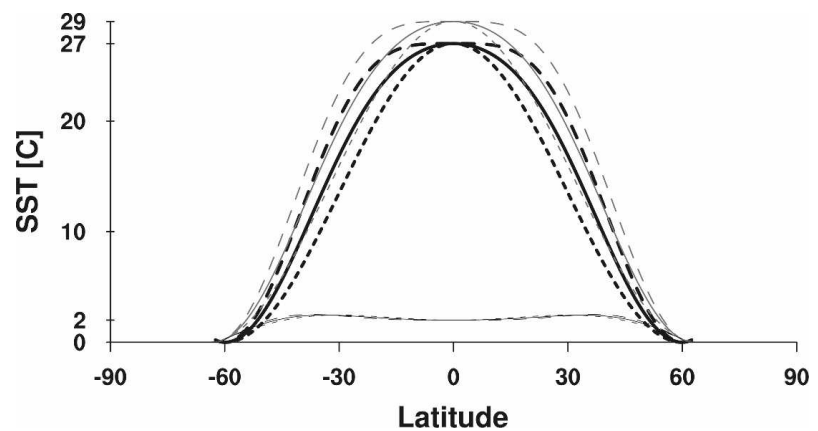

FIG. 1. SST profiles for the APE (black) and SST +2 (gray) aquaplanets. The cases are described as A (short dashed), B (solid), and C (long dashed). Difference curves (SST +2 minus APE) are also shown as thin curves, with the same dash patterns as the SST profiles.

To make the aquaplanets used here as uniform as possible, the conventions of the Aqua-Planet Experiment Project (APE; Neale and Hoskins 2001) are followed. Three SST profiles for baseline simulations also use APE conventions; they are simple analytical forms with maximum SST on the equator, falling to $0^{\circ} \mathrm{C}$ in the high latitudes. These APE SST profiles, shown as solid curves in Fig. 1, are given as functions of latitude $\phi$ by

$$
\begin{aligned}
& T_{A}(\phi)=T_{\max }\left[1-\sin ^{2}\left(k_{1} \phi\right)\right] \\
& T_{B}(\phi)=0.5 T_{\max }\left[2-\sin ^{2}\left(k_{1} \phi\right)-\sin ^{4}\left(k_{2} \phi\right)\right] \\
& T_{C}(\phi)=T_{\max }\left[1-\sin ^{4}\left(k_{2} \phi\right)\right],
\end{aligned}
$$

where $T_{\max }$ is the maximum SST (realized on the equator), and $k_{1,2}$ are parameters determining the latitude where SST reaches $0^{\circ} \mathrm{C}$. For simplicity, the APE nomenclature for the SST profiles is replaced by "A," "B," and "C" for progressively smaller tropical SST gradients (hence, more baroclinic extratropics). Table 1 provides the APE names for convenience. The APE conventions assign $T_{\max }=27^{\circ} \mathrm{C}$ and $k_{1,2}=1.5$.

This analysis is chiefly centered on idealized climate change experiments performed by assigning warmer SST to the aquaplanets, shown as gray curves in Fig. 1 and also given by Eq. (1). Such a framework for evaluating climate sensitivity has been used extensively in the past (e.g., Cess et al. 1989, 1990, 1996, and many others), lends itself well to aquaplanet studies, and, as shown by Ringer et al. (2006), appears to capture some essential aspects of the climate sensitivity of more realistically forced simulations. For the warmer $(\mathrm{SST}+2)$ simulations, $T_{\max }=29^{\circ} \mathrm{C}$. Because only changing $T_{\text {max }}$ would focus the temperature change on the equator, $k_{1}$ and $\mathrm{k}_{2}$ are also varied so as to yield the flattest possible temperature difference between the APE and SST +2
TABLE 1. Description of aquaplanet configurations in terms of APE. Note S stands for the standard, Earth-like boundary conditions with realistic geography and climatological SST.

\begin{tabular}{cl}
\hline \hline Name & APE \\
\hline S & N/A \\
A & Control \\
B & Qobs \\
C & Flat \\
\hline
\end{tabular}

simulations within the latitude band of $\phi= \pm \pi / 3$. The resulting values for $k_{1}$ and $k_{2}$ are 1.407 and 1.439 , respectively, and the difference curves are included in Fig. 1. The idea of limiting the SST increase to the tropics was motivated by a desire to avoid unrealistically large temperatures near the poles. Limited calculations with uniform surface temperature perturbations show that the tropical response, which we focus on here, is not sensitive to whether or not the temperatures in the extratropics are also increased. In retrospect, such uniform surface temperature perturbations would have been a preferable basis for all of our analysis.

In the following sections, attention is focused on the tropics, but there is some ambiguity associated with choosing latitudes to represent the tropical atmosphere. The width of the Hadley circulations varies among the configurations. To make matters more complicated, when SST is increased the Hadley circulation generally extends meridionally; tropical convergence zones typically move one grid point poleward, for example. In the following, the tropical belt is defined as the region bounded by $35^{\circ} \mathrm{S}$ and $35^{\circ} \mathrm{N}$, because this captures all or most of the descending branch of the Hadley circulation for all configurations considered. Analysis using objective definitions of the tropics, either based on the vertically integrated streamfunction or the latitude at which surface winds switch from easterlies to westerlies, have shown qualitatively similar results.

Combining simulations using the original APE SST profiles and those with SST +2 profiles gives three aquaplanet climate change experiments for each GCM. These aquaplanet experiments are compared with similar experiments using standard, Earth-like configurations of each GCM with uniform, global increase of $2 \mathrm{~K}$ in SST; throughout the text these more realistic simulations are referred to interchangeably as either standard or Earth-like. To test for possible sensitivity to horizontal resolution, all experiments are performed with the NCAR CAM at T42 and T85 truncations (both with the default Eulerian dynamical core). These resolutions yield grid spacings of $2.8^{\circ} \times 2.8^{\circ}$ and $1.4^{\circ} \times 1.4^{\circ}$, respectively. The GFDL AM grid size is $2^{\circ}$ latitude $\times$ $2.5^{\circ}$ longitude. Following APE conventions, the aqua- 

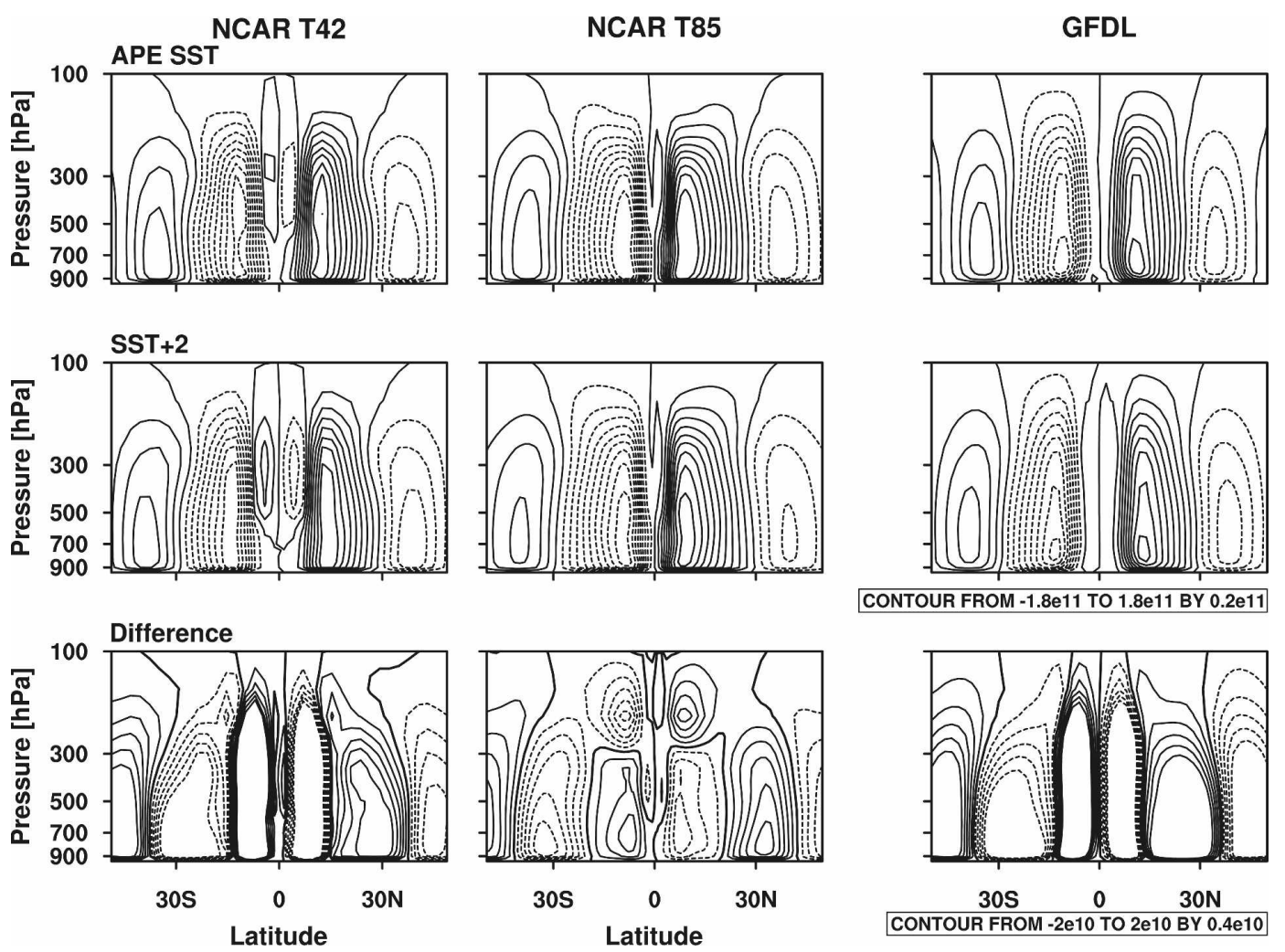

FIG. 2. Mean meridional circulation, as illustrated by the meridional streamfunction $\left(\mathrm{kg} \mathrm{s}^{-1}\right)$. The columns are the different GCMs, all with the intermediate (top) APE SST distribution (aquaplanet B) and (middle) SST +2 configurations. (bottom) The difference in the streamfunction between SST +2 and APE SST is shown. The horizontal axis runs from $-50^{\circ}$ to $50^{\circ}$. Contour intervals are included on the right side of figure, but also note that for the MMC, the T85 version of NCAR CAM uses the full contour interval while the other two configurations use one less contour: from $-1.6 \times 10^{11}$ to $1.6 \times 10^{11} \mathrm{~kg} \mathrm{~s}^{-1}$; all three difference panels use the full contour interval.

planet simulations are all integrated for 42 months, with the last 36 months used in the analysis. To yield stable statistics, the standard configurations of the GCMs require longer integrations, so their analysis is based on averages taken over the last 60 months of integration. Unless otherwise noted, all analysis is based on monthly mean output.

\section{Aquaplanet climate}

As a prelude to the discussion of climate sensitivity and cloud effects, this section reviews the basic character of the aquaplanet simulations and how they respond to a change in their underlying SSTs.

In all three sets of simulations, strong Hadley circulations rise in the deep tropics and subside in neartropical latitudes. This is evident in Fig. 2, which shows the mean meridional circulation (MMC) from simulations using the intermediate APE SST profile (aquaplanet B) and the SST +2 version. This MMC is stronger and generally more tropically confined than in re- alistic simulations or observations. The location and strength of each branch varies with GCM and SST, but the overall structure is qualitatively similar. Poleward of the Hadley cells, thermally indirect Ferrel cells are evident. The response of the circulation to an increase in SST is discussed below. The degree of hemispheric symmetry in the figure gives a sense of the statistical convergence achieved, giving confidence that the averaging interval $(3 \mathrm{yr})$ is adequate.

Figure 3 presents the zonal mean precipitation rate for all the aquaplanet simulations, showing the hydrological expression of circulations like those in Fig. 2. Because of the strong hemispheric symmetry, the hemispheres are averaged here. Every aquaplanet exhibits tropical precipitation organized into tropical convergence zones, associated with regions of strong ascent in Fig. 2. Dry zones are associated with the descending branch of the Hadley cells. The secondary precipitation maxima poleward of the dry zones represent the midlatitude storm tracks.

As the region of warmest SST becomes extended me- 

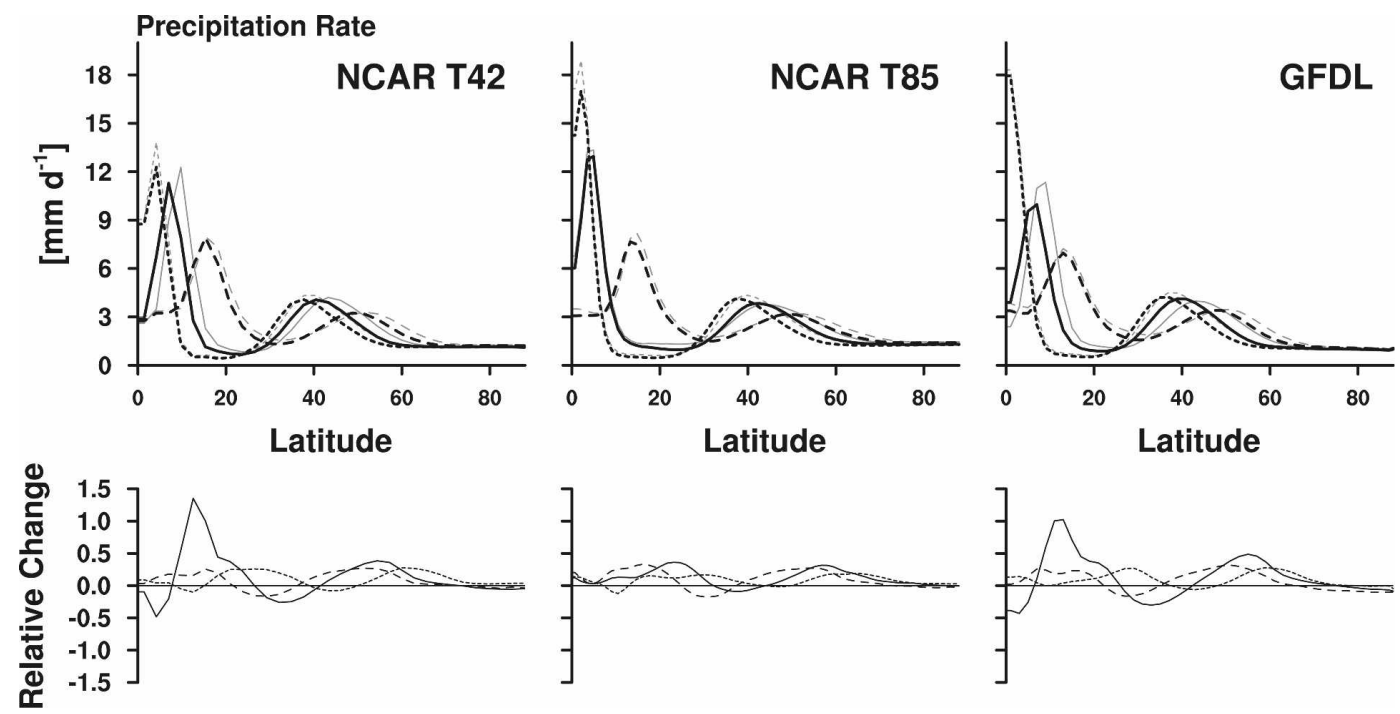

FIG. 3. Zonal average, time mean total precipitation rate from all the aquaplanet simulations. Black curves in the top row are the simulations using APE SST, and the gray curves are the SST +2 cases; dash patterns are as in Fig. 1. The lower row shows the change relative to the APE SST simulation for each configuration. The hemispheres are averaged about the equator, so the curves run from equator to pole.

ridionally, the tropical convergence zones straddling the equator become well separated, and the maximum precipitation decreases (as shown in Fig. 3). When the SST is especially peaked (aquaplanet A; short dashed lines in Fig. 3), there is a tendency for the two ITCZs to merge, or nearly merge, at the equator. Midlatitude storm tracks are also affected by the size of the tropics; as the tropics expand, the local maximum precipitation rate decreases and the storm track becomes more meridionally extended. Such changes are associated with small, but not qualitative, differences in the implied meridional ocean heat transports among the simulations. Overall, the family of circulations given by variations in the underlying distribution of SST serves the purpose of sampling a range of modestly Earth-like conditions and helps us address the extent to which cloud responses to warming are robust to changes in the control circulation.

\section{Warming the aquaplanets}

The tendency of the precipitation rates in the tropical rainbelts to increase in the SST +2 simulations compared to the APE SST simulations is consistent with the robust response of more realistically configured climate models (Held and Soden 2000, 2006). In the extratropics the difference is smaller, with the most substantial change being a poleward shift of the midlatitude storm track.

Differences in zonal mean precipitation are associated with changes in the large-scale circulation. In each case the warmer SST induces an increase in the meridional extent of the Hadley circulation. This is especially evident in aquaplanet B of the T42 version of NCAR CAM and GFDL AM as poleward shifts in the ITCZ. The T85 simulation, however, shows a more pronounced change in the vertical extent of the rising branch of the Hadley circulation, with a smaller poleward migration of the ITCZ. Both effects manifest in all the aquaplanets, but the balance of the two changes can be very different, as shown by the difference panels in Fig. 2. The poleward shift of the tropical MMC corresponds with a relative decrease in precipitation on the equatorward side of the ITCZ and an increase on the poleward side. The change in the vertical extent of the circulation appears as a relative increase in precipitation across the ITCZ (Fig. 3). Surprisingly, the T42 version of the NCAR CAM exhibits a response more similar to the GFDL AM than to the higher-resolution T85 version.

Figures 2 and 3 suggest a dependence of the meridional circulation (and processes related to it) on model resolution. Williamson and Olson (2003) reported that NCAR CAM aquaplanets obtain very different zonal mean precipitation when the dynamical core is changed from Eulerian to semi-Lagrangian. The Eulerian version was run at T42, as in the left column of Fig. 3, but with a previous version of the Community Climate Model. At that time, the difference arose from a dependence on time step, which affected how far moisture was carried toward the equator after evaporating from 
the surface. It is unclear what causes the difference in the response to increased SST in the current version.

\section{Climate sensitivity}

The simulations above constitute climate change experiments similar to those of Cess et al. (1989, 1990, 1996) and many others. By artificially changing SST, the top-of-atmosphere (TOA) energy budget is altered, so changes in TOA radiative fluxes are typically used to quantify the climate response. Climate sensitivity is measured using the climate sensitivity parameter $\lambda$, following Cess et al. $(1989,1990)$, defined as the difference in surface temperature $T_{s}$ between simulations for a given radiative forcing $\lambda=\Delta T_{s} / G$, where $G=\Delta Q-$ $\Delta F$ and $Q$ and $F$ are the net longwave and shortwave fluxes at TOA and $\Delta$ is the difference between the simulations. The cloud effect parameter is defined by the ratio of the all-sky to clear-sky sensitivity, and is related to the change in cloud radiative forcing (CRF) and direct radiative forcing, $\lambda / \lambda_{\text {clr }}=(\Delta \mathrm{CRF} / G)+1$. Although the literature tends to associate $\Delta \mathrm{CRF} / G$ with cloud feedbacks, this need not be the case; by construction, $\Delta \mathrm{CRF} / \mathrm{G}$ can be nonzero even if clouds do not change (Soden et al. 2004).

Figure 4 shows a comparison of $\lambda$ and $\Delta \mathrm{CRF} / G$ for the tropics for all the experiments. The values are calculated using a tropical belt between $35^{\circ} \mathrm{S}$ and $35^{\circ} \mathrm{N}$, as discussed in section 2. All NCAR CAM configurations (denoted with "N") are tightly clustered in Fig. 4, with negative values of $\Delta \mathrm{CRF} / G$ and low values of $\lambda$. The GFDL AM configurations (denoted with "G") are all more sensitive (large values of $\lambda$ ), with positive values of $\Delta \mathrm{CRF} / G$. The standard configurations $\left(N_{S}, N_{S}^{+}, G_{S}\right)$ have values of $\lambda$ and $\Delta \mathrm{CRF} / G$ similar to the aquaplanets from the same GCM. For comparison, the globally averaged values of the standard, Earth-like configurations are shown in gray. Overall, the tropically averaged response is in the same sense as the global response, albeit slightly exaggerated, with small differences arising from extratropical effects. The globally averaged values from the aquaplanets are omitted because no climate change is prescribed at high latitudes.

Taken as a whole, Fig. 4 suggests the aquaplanet configuration captures much of the relationship between cloud radiative response and climate sensitivity at low latitudes. The clustering of values suggests that changing from the standard configuration to aquaplanets, or changing the horizontal resolution, has little influence on sensitivity or cloud forcing changes. The GFDL AM response, especially in $\Delta \mathrm{CRF} / G$, shows more sensitivity to the definition of the tropical domain over which averages are taken, and thus the characteristics of the

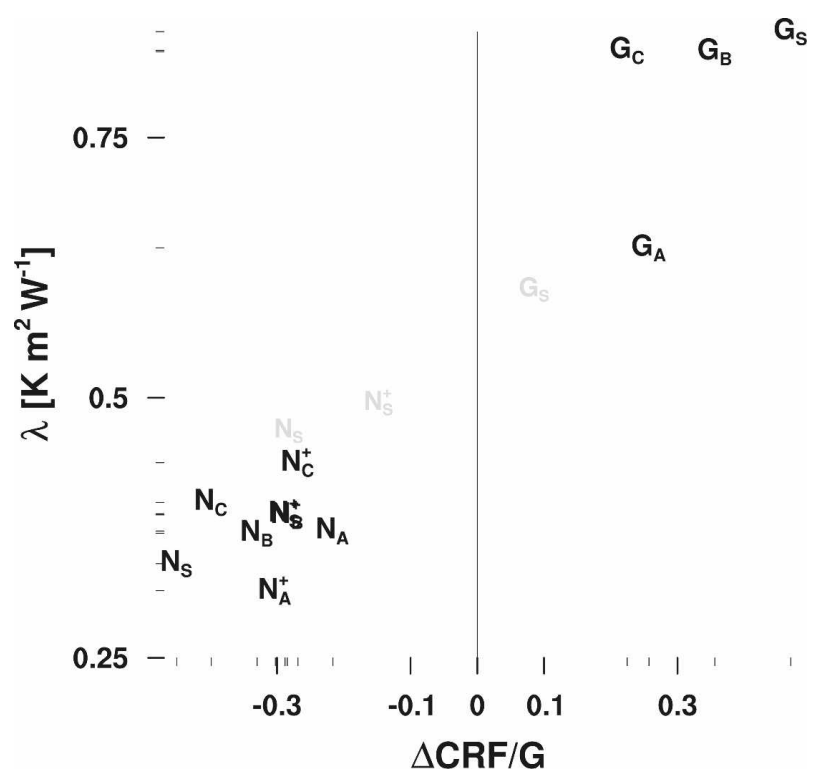

FIG. 4. Climate sensitivity parameter $\lambda$ vs $\Delta \mathrm{CRF} / G$ for all configurations of both GCMs; black symbols represent the tropically averaged values. The symbols correspond to abbreviations of each configuration, where the large letter gives the GCM being used ( $\mathrm{G}=$ GFDL AM, N = NCAR CAM), the subscript is the SST configuration ( $\mathrm{S}$ for standard, $\mathrm{A}-\mathrm{C}$ for aquaplanets), and a superscript "+" denotes the T85 version of the NCAR CAM. The two nearly overlain symbols are the T85 versions of the standard and aquaplanet B configurations. Gray symbols denote the globally averaged values from the standard configurations.

large-scale circulation, than does the NCAR CAM. However, the link between climate sensitivity and cloud response seems to persist even for the least Earth-like aquaplanets. For example, aquaplanet $\mathrm{C}$ for the GFDL AM hints at an ostensible third tropical convergence zone (on the equator) in the SST +2 case (Fig. 3), but still shows an overall cloud response and climate sensitivity in the tropics close to the standard case.

The clear-sky climate sensitivity $\lambda_{\text {clr }}$ has also been investigated. The aquaplanets from both GCMs produce tropical $\lambda_{\text {clr }}$ within a range half the size of that for $\lambda$, from 0.45 to $0.68 \mathrm{Km}^{2} \mathrm{~W}^{-1}$ compared with $0.31-0.85$ $\mathrm{Km}^{2} \mathrm{~W}^{-1}$. The standard configurations show even less spread in $\lambda_{\text {clr }}$, with values of $0.54,0.58$, and $0.62 \mathrm{~K} \mathrm{~m}^{2}$ $\mathrm{W}^{-1}$ for NCAR CAM T85, GFDL AM, and NCAR CAM T42, respectively. The reason why the spread in clear-sky flux response is greater in the aquaplanets than in the standard configuration remains to be determined. The effects of clouds are largely responsible for variations in climate sensitivity, especially in the tropics.

In summary, the two GCMs respond quite differently for the same prescribed climate change, and the aquaplanets respond similarly to the standard configurations for both GCMs. These results support the hypothesis 
that aquaplanets capture much of the standard GCM's tropical climate response to an idealized SST perturbation. It appears that aquaplanets have a tendency to mirror the divergent response of the parent GCMs, rather than converging to a single, common solution.

\section{Cloud changes conditioned on dynamical state}

From Fig. 4 it appears that, like the more Earth-like configurations, the climate sensitivity $\lambda$ of the aquaplanets is linked to changes in CRF. The aquaplanets have a variety of large-scale circulations, which raises the question as to whether their cloud response, as measured by $\triangle \mathrm{CRF}$, is more than superficially similar to the cloud response in the Earth-like simulations. If so, it suggests that the same physical processes responsible for the climate response in Earth-like settings are also at work in the aquaplanets.

To begin answering this question we use the framework introduced by Bony et al. (2004), where changes in the cloud radiative forcing are conditioned on the dynamical state,

$$
\begin{aligned}
\Delta \overline{\mathrm{CRF}}= & \int P_{\omega} \Delta \mathrm{CRF}_{\omega} d \omega \\
& +\int \mathrm{CRF}_{\omega} \Delta P_{\omega} d \omega+\int \Delta \mathrm{CRF}_{\omega} \Delta P_{\omega} d \omega .
\end{aligned}
$$

The lhs is simply the tropically averaged difference in CRF; $P_{\omega}$ is the probability a location has vertical motion $\omega$ (equivalently, the pdf of the dynamic variable $\omega$ ), $\mathrm{CRF}_{\omega}$ is the CRF as a function of $\omega$, and $\Delta$ is again the difference between the SST +2 and APE case. By convention the APE SST simulation is taken as the baseline, providing $P_{\omega}$ and $\mathrm{CRF}_{\omega}$. The rhs terms are associated with changes in CRF for a given dynamical state (thermodynamic effect), changes in the large-scale circulation (dynamic effects), and covariations of dynamic and thermodynamic influences, respectively. Using vertical motion at $500 \mathrm{hPa}\left(\omega_{500}\right)$ to define the dynamical state, Fig. 5 shows the tropically averaged change in CRF along with the three terms of Eq. (2). The total change (black bars) is consistent with the changes in cloud forcing in Fig. 4. The thermodynamic component (red bars) is the largest contribution in all but one case, and dominates the NCAR CAM simulations. The small dynamic components in all of these tropical averages are sensitive to the choice of tropical area.

The tendency of changes in the CRF to be associated with the thermodynamic component of the response has been used to argue that the cloud response is likely determined mainly by the parameterized physics (Bony and Dufresne 2005; Wyant et al. 2006). The fact that the character of this response is robust to the details of the circulation provides further support for this idea.

\section{Clouds}

Determining whether the physical processes responsible for the climate response with Earth-like conditions are also at work in the aquaplanets requires a deeper analysis of the relation between the cloud response in the different configurations. To begin delving into this issue, Fig. 6 shows tropical cloudiness and how it changes as SSTs are warmed for each set of simulations, decomposed into high-, middle-, and low-level clouds; total cloudiness is also shown. Letters mark the tropically averaged values from the standard, Earthlike GCM experiments, while connected circles indicate the range covered by the three aquaplanets. It should be noted that each category of cloud is separately diagnosed by each GCM using different methods of vertical integration (i.e., different cloud overlap assumptions). Cloud categories are determined using fixed pressure levels, middle clouds are bound by 700 and 400 $\mathrm{hPa}$ in the NCAR CAM and 680 and $440 \mathrm{hPa}$ for the GFDL AM; because of the coarse vertical resolution of both GCMs, the difference between the definitions of the cloud categories is negligible compared to the differences in cloud schemes.

High-cloud coverage decreases in most experiments. While the longwave (warming) and shortwave (cooling) effects of high clouds are known to nearly cancel (Kiehl 1994), a slight imbalance tends to tilt in favor of warming, so the decrease in high-cloud amount in Fig. 6 is usually considered a negative radiative feedback (less warming). In both GCMs, this would suggest that the net radiative effect of high clouds is weak and negative, while the change in CRF is opposite in sign between the two GCMs. Middle-level clouds are much less pervasive than high or low clouds in both models, and also show little consistency in their response, suggesting they too are not of leading order in determining the overall cloud response or climate sensitivity.

The albedo effect of low clouds dominates their greenhouse effect, so an increase in low-cloud amount is associated with a decrease in cloud radiative forcing. Among the simulations, only the change in low clouds is consistent with the change in cloud radiative forcing across the tropics. Moreover, the sign of the total cloud change matches that of low clouds in each configuration. Both tend to support the idea that differences in the simulated climates are largely a result of differences in the representation of low clouds (e.g., Bony and Dufresne 2005). 

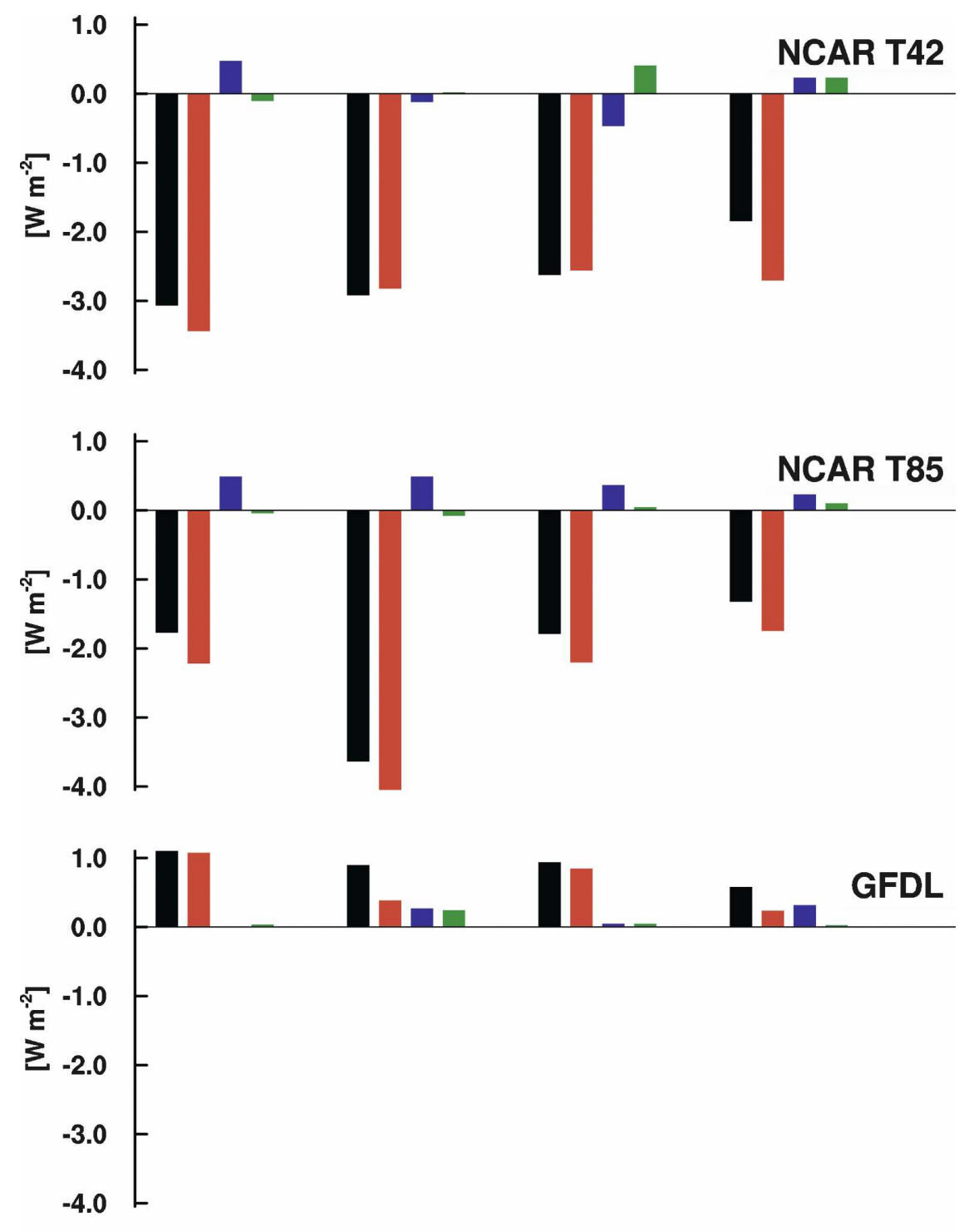
S
A
B
C

FIG. 5. Decomposition of the change in CRF. Each panel shows a separate GCM configuration, that is, the two horizontal resolutions of the NCAR CAM and the GFDL AM. Within each panel, four sets of bars are shown, one for each configuration (noted at the bottom). (left to right) The bars are the tropical average CRF change (black), thermodynamic contribution (red), dynamic contribution (blue), and covariation contribution (green).

Directly comparing $\lambda$ and the change in low-level clouds substantiates the notion that low clouds are an essential control on climate sensitivity. Figure 7 shows the comparison, in analogy to Fig. 4, demonstrating the dramatic difference between the GFDL AM and NCAR CAM in low-cloud response. There is a tendency for lower climate sensitivity with larger increases in low-cloud amount. Figure 6 shows that aquaplanets do well at predicting low-cloud amount as well as the change in low clouds associated with the uniform SST increase in the standard configuration, explaining why aquaplanets are successful predictors of climate sensitivity in these experiments.

\section{Dynamical regimes}

The analysis presented in section $4 \mathrm{a}$ attempts to untangle changes in cloudiness resulting from changes in the circulation (or dynamical state, here measured by $\omega_{500}$ ) from changes that occur for a given circulation (or dynamical state). Previous studies have found the 


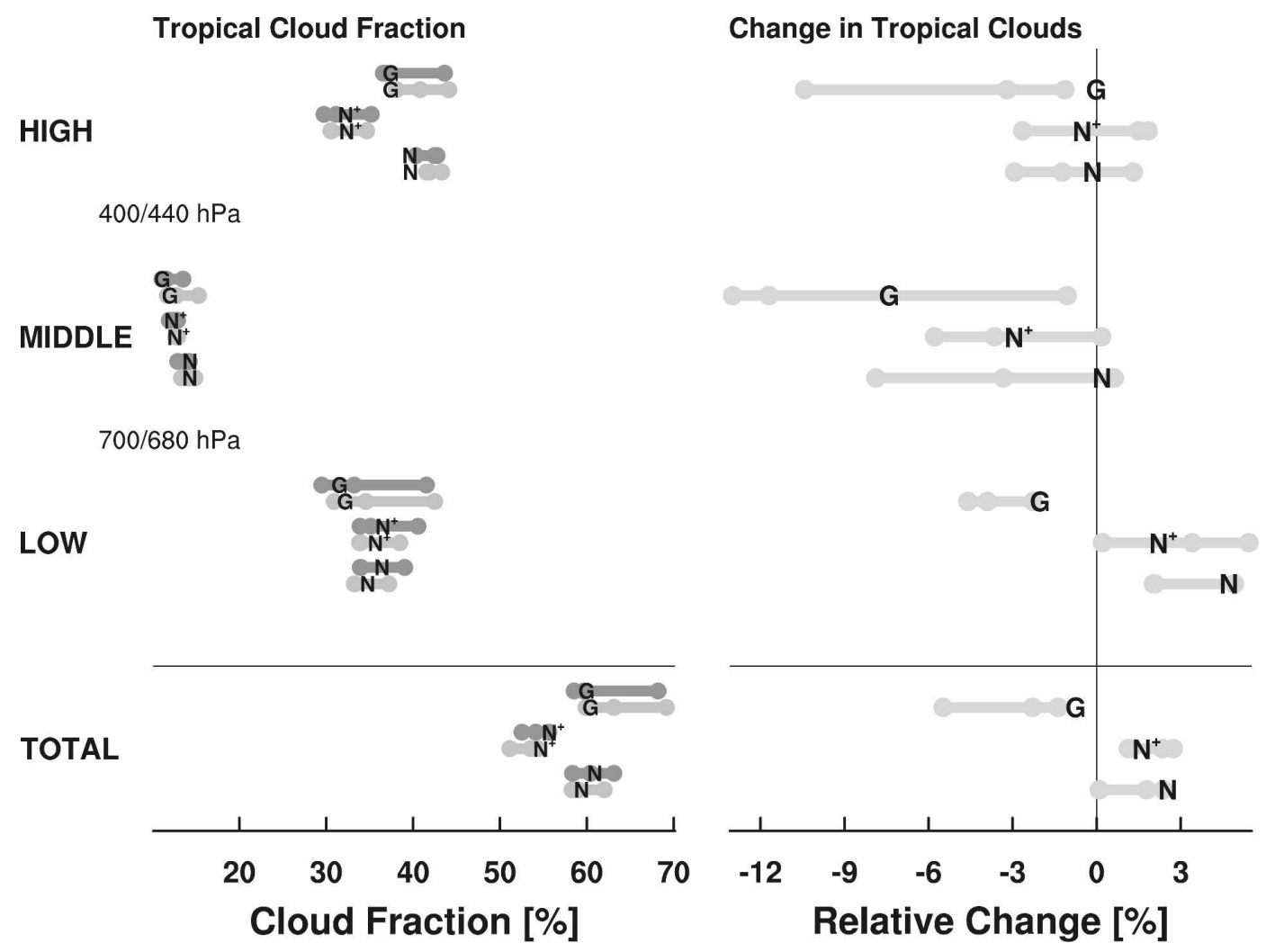

FIG. 6. Tropical cloud fraction and relative change, for high, middle, and low clouds, and with the total cloud fraction at the bottom (below horizontal line). (left) Area-weighted tropical mean cloud fraction from the Earthlike GCM configurations as letters $\left(\mathrm{N}, \mathrm{N}^{+}\right.$, and $\left.\mathrm{G}\right)$, and aquaplanets for each as gray circles connected by horizontal lines; aquaplanets are not distinguished here as A, B, and C. The APE SST configuration (lighter gray) is below the SST +2 results (darker gray) for each GCM. (right) Change in cloud fraction relative to the APE SST distribution, again using characters for the Earth-like configuration and connected circles to show the range of cloud changes in the aquaplanets. Pressure levels differentiating the categories are noted on the left panel: 700 and $400 \mathrm{hPa}$ for NCAR CAM and 680 and $440 \mathrm{hPa}$ for GFDL AM.

strongest contribution to the tropics-wide response is concentrated in regions of large-scale subsidence (e.g., Bony et al. 2004), consistent with our finding that changes in low clouds are the best indicator of the overall behavior of the different simulations. To address whether or not the aquaplanets behave similarly to the Earth-like simulations in this respect, we aggregate the terms of Eq. (2) over ranges of the dynamic variable to construct qualitative dynamical "regimes." Changes within each regime can be decomposed analogously to the procedure used to analyze the tropics as a whole, for example, Eq. (2),

$$
\begin{aligned}
\Delta \overline{\mathrm{CRF}\left(\mathbf{S}_{\mathbf{i}}\right)}= & \sum_{\omega_{i} \leq \omega<\omega_{i+1}} P_{\omega} \Delta \mathrm{CRF}_{\omega} \\
& +\sum_{\omega_{i} \leq \omega<\omega_{i+1}} \operatorname{CRF}_{\omega} \Delta P_{\omega} \\
& +\sum_{\omega_{i} \leq \omega<\omega_{i+1}} \Delta \mathrm{CRF}_{\omega} \Delta P_{\omega} .
\end{aligned}
$$

Here $\mathbf{S}_{\mathbf{i}}$ denotes the regime, defined as the set of locations with $\omega$ in the interval defined by the index $i, \mathbf{S}_{\mathbf{i}}=$ $\left\{\omega \mid \omega_{i} \leq \omega<\omega_{i+1}\right\}$. Figure 8 shows a set of these regimes, organized into several categories to capture gradations of convective or subsiding motion; the intervals used here are equally spaced with an interval of $30 \mathrm{hPa}$ day $^{-1}$, and the dynamic variable is again the pressure velocity at $500 \mathrm{hPa}\left(\omega_{500}\right)$. The figure shows the contribution to the total change in CRF of each set of regimes, so summing across regimes for any configuration gives the values of the black bars in Fig. 5 .

Figure 8 indicates that the similarity between the standard, Earth-like configuration (black) and the aquaplanets also holds within dynamic regimes. Concentrating on the subsidence regimes, which contribute the most to changes in cloud radiative forcing, we note that here again the aquaplanet version of a model behaves more similarly to the Earth-like version of that same model than to an identically configured aqua- 


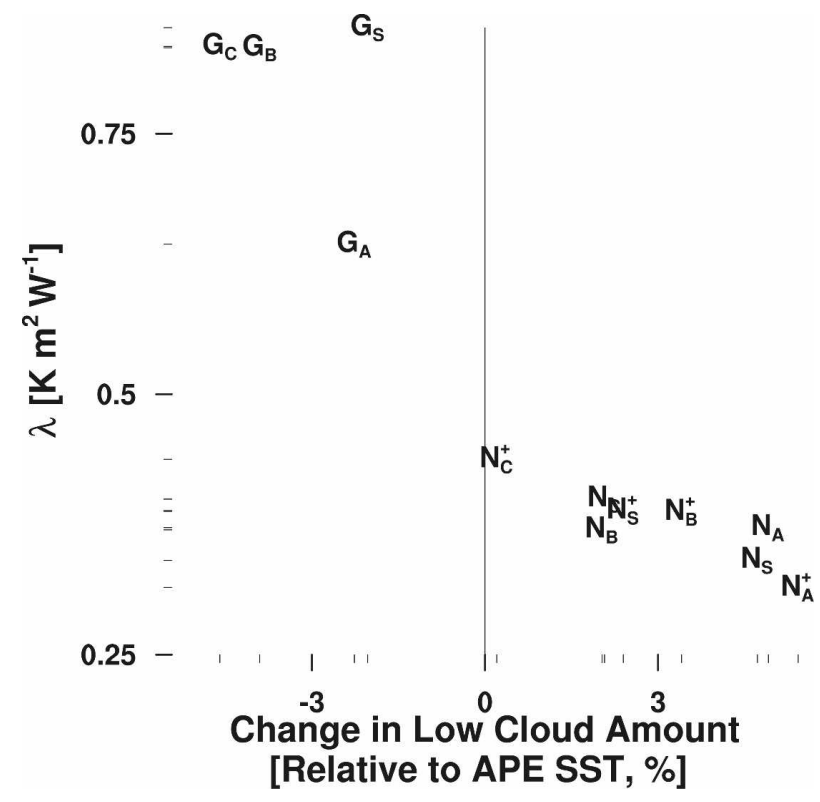

FIG. 7. Same as Fig. 4, but for tropical low-cloud fraction and climate sensitivity parameter. The change in low-cloud fraction is relative to the APE SST baseline case, the actual change is small, as demonstrated by Fig. 6 .

planet simulation by another model. Essentially every simulation shows a decrease in (negative) cloud radiative forcing in the weak subsidence regime and an increase in the strong subsidence regime. The difference between the models is that changes in the strong subsidence regime carry the day for the case of the GFDL AM experiments, whereas changes in the weak subsidence regime prevail in the NCAR CAM experiments. The NCAR CAM aquaplanet $\mathrm{C}$, the flattest tropical SST distribution, is an exception, because it is most influenced by stronger (negative) CRF in weak convective regions. That exception helps illustrate that aquaplanets are not identical to the Earth-like configurations; there are differences because the large-scale circulations are quantitatively different, leading to slightly different climate responses. The differences can be slightly exaggerated (or diminished) by choice of the tropical area to use in averaging, as mentioned above. The qualitative behavior of Earth-like and aquaplanet configurations is similar though, especially considering the response in different regimes, the contribution of each regime to the total change in CRF, and the division between thermodynamic and dynamic components, as discussed next.

The thermodynamic and dynamic contributions for each experiment are included in Fig. 8 as circles and squares, respectively. The terms tend to cancel for regimes with a small change in CRF, which is especially apparent for the strong convection regime. On the other hand, regimes where the two contributions act in the same sense tend to have the strongest influence on the CRF response. The figure demonstrates that dynamic and thermodynamic contributions can both play roles in particular regimes, similarly for aquaplanets and Earth-like configurations. Based on an analysis of three realistically configured GCMs, Bony et al. (2004) similarly noted that regional changes in CRF are strongly influenced by changes in circulation while tropically averaged changes are mostly due to thermodynamic effects. Wyant et al. (2006) present one interpretation of the difference between tropical averages and individual regimes, noting that the dynamic term of Eq. (2) might be expected to be small when the domainaveraged $\omega$ is small. This is the case for the tropically averaged vertical motion. However, as we define them, individual regimes can cover large areas of the tropics, but are characterized by nonvanishing $\bar{\omega}$ by construction.

Although the dynamic terms contribute to the climate response in the models, Fig. 8 shows that it is generally the thermodynamic component of the response that behaves differently between the NCAR CAM and the GFDL AM, irrespective of the details of the forcing. That is, the contribution from the change in circulation, shown by squares in Fig. 8, is more consistent across all configurations than the thermodynamic contribution (circles), which tends to be similar within a set of GCM configurations, but not necessarily across the GCMs. This disagreement is most apparent in the weak subsidence regime in which the GFDL AM shows a tendency for decreases in cloud fraction, leading to an increase in CRF, while the NCAR CAM experiments show the opposite: stronger, more negative CRF associated with enhanced cloud cover. The fact that the aquaplanets capture this aspect of the response of the more Earth-like simulations suggests that the same physical processes are responsible for the climate response of both configurations.

\section{Discussion}

To begin developing some insight into how the cloud responses of the models differ as a function of the thermodynamic state, we construct joint histograms of lower-tropospheric stability [using the conventional measure, $\left.\theta(p=700 \mathrm{hPa})-\theta\left(p=p_{\text {sfc }}\right)\right]$ and low-cloud amount for the standard configuration and one aquaplanet (B) for both GCMs. The joint histograms, shown in Fig. 9, are separated into four dynamical regimes based on $\omega_{500}$, corresponding to Fig. 8; data are contoured so that summation across lower-tropospheric stability, low-cloud amount, and dynamical regimes re- 

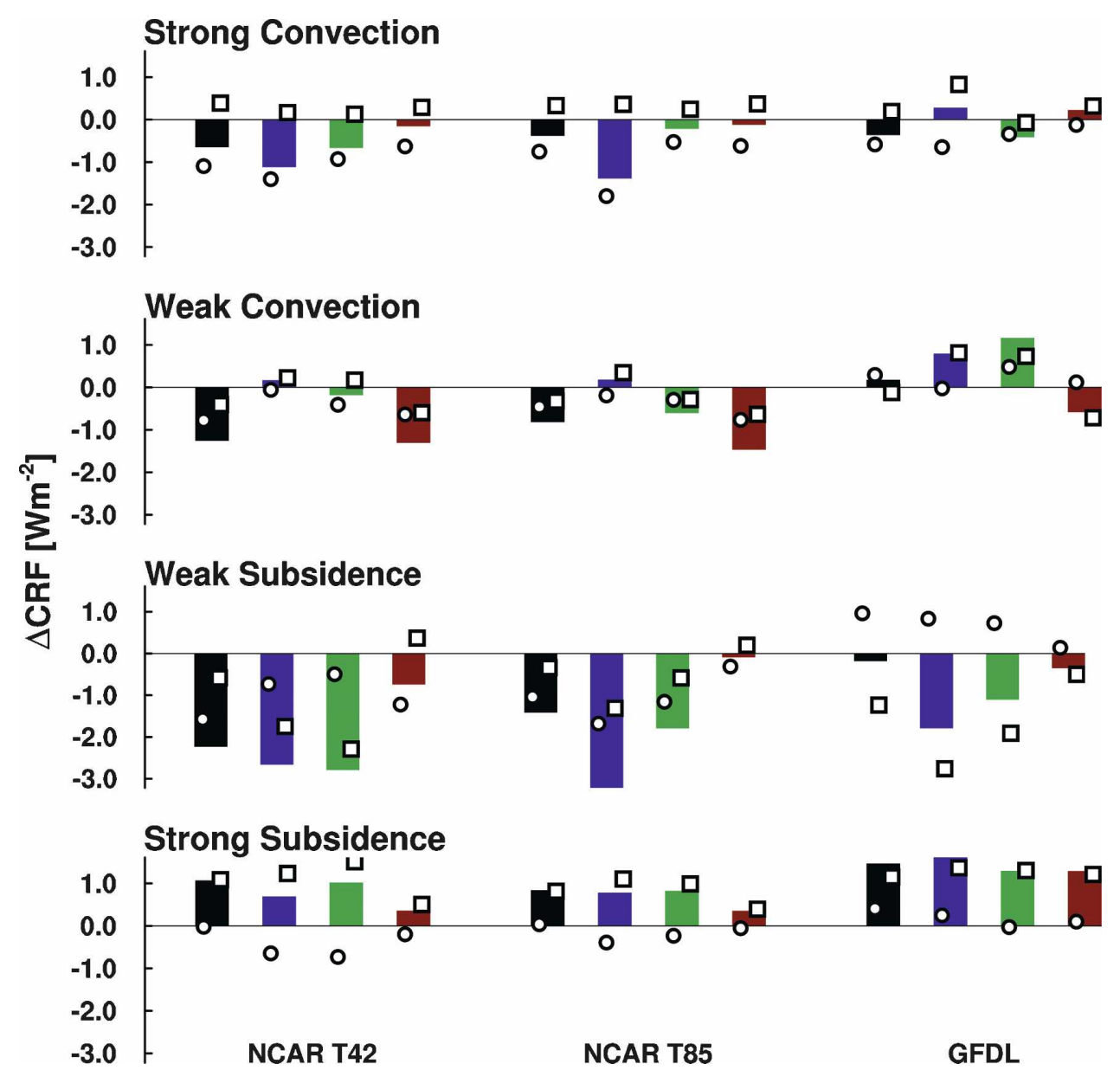

FIG. 8. Tropical CRF change for all experiments, sorted into dynamical regimes. (top to bottom) The regimes are strong and weak convective regimes and then weak and strong subsidence regimes. Within each regime three sets of bars represent each GCM (NCAR CAM at T42 and T85 and GFDL AM, respectively, noted at bottom). The bars for each GCM are the standard, Earth-like configuration (black) followed by the three aquaplanets: A (blue), B (green), and C (red). The thermodynamic and dynamic terms for each experiment are marked with circles and squares respectively.

turns unity, that is, fewer contours in a panel reflect less data within that regime. The difference in low-cloud amount between the APE SST (black) and SST +2 (red) simulations within each regime essentially captures the thermodynamic influence from Eq. (2). The dynamic term is a change in the pdf of $\omega_{500}$, which is largely related to changes in the area covered per regime (the fraction of the tropics covered by each regime is also reported in Fig. 9). The distribution of lowertropospheric stability is an expression of the large-scale environment within these qualitative regimes; generally low-cloud amount is thought to increase with lowertropospheric stability, as the strength of the trade inversion increases (e.g., Slingo 1980; Klein and Hartmann 1993). Lower-tropospheric stability is, however, a fairly crude measure of inversion strength, and more elaborate measures have recently been shown to better correlate with observed low-cloud amount (Wood and Bretherton 2006); however, their chief benefit is in extratropical regions, which we do not consider here.

In response to increased SST, the distributions in Fig. 9 show a robust shift toward higher stability, which is especially evident in the means. This shift toward higher stability indicates a larger temperature response at $700 \mathrm{hPa}$ than at the surface. Although both models show similar changes in stability, the changes in lowcloud amount within regimes differ: the NCAR CAM configurations have increases in low cloud in subsidence regimes, while the GFDL AM configurations change in the opposite sense, with the largest changes in the weak convective and weak subsidence regimes. In this respect the behavior of the NCAR CAM falls in line with prevailing thought that low-cloud amount increases with increasing stability. 

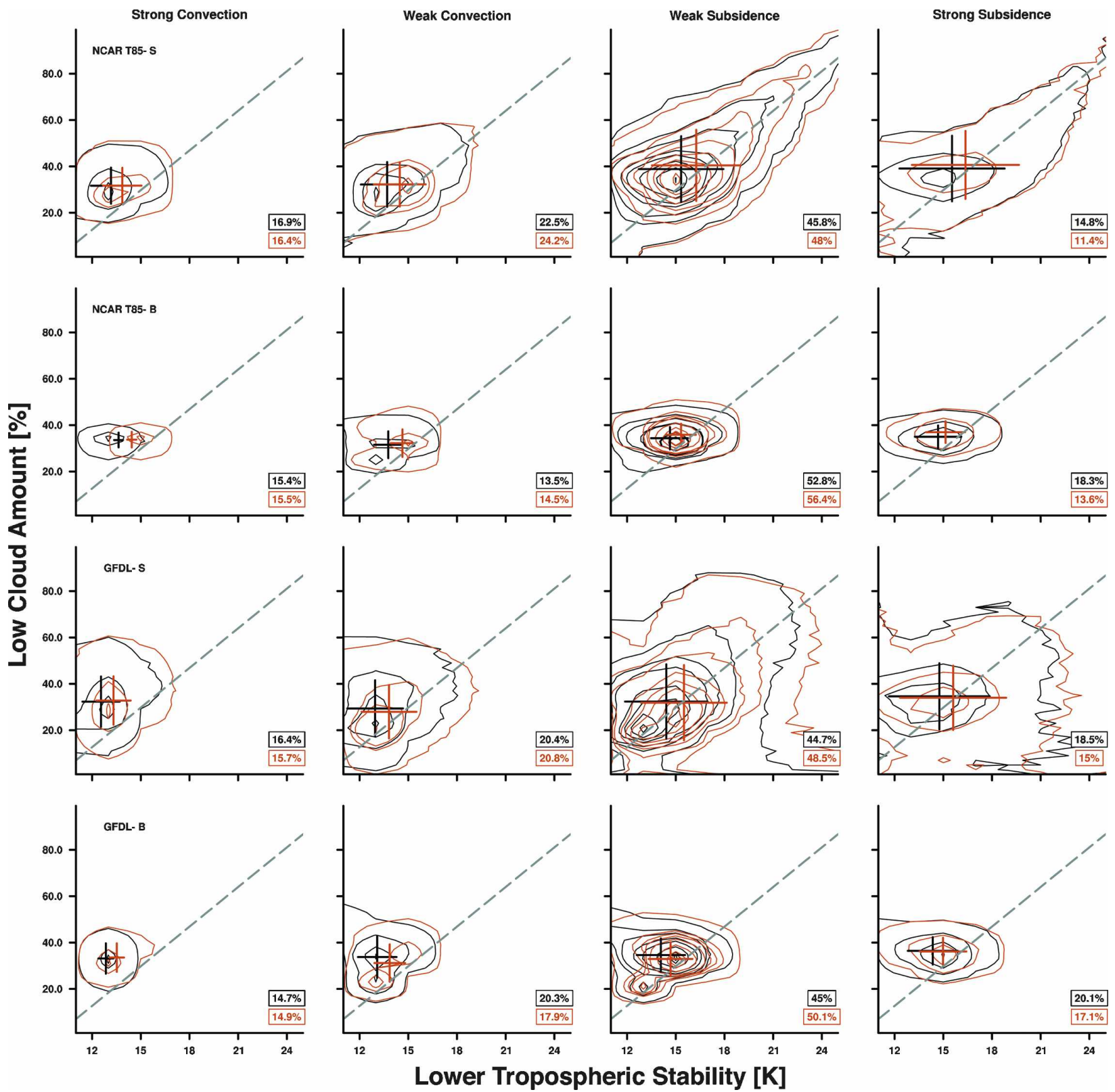

FIG. 9. Joint histograms (units of probability) of lower-tropospheric stability and low-cloud amount for the T85 version of the NCAR CAM and the GFDL AM for the standard configuration (S) as well as aquaplanet B. Columns represent ranges of vertical pressure velocity at $500 \mathrm{hPa}$. Rows are the different model configurations, noted at the upper left of the left column. The contours show the likelihood of low-cloud amount and lower-tropospheric stability values in the tropics; the smallest contour for each panel corresponds to $1 \%$ of the total tropical belt and the most likely values of stability and low-cloud amount; larger contours increase by $6 \%-43 \%$ (i.e., the contour interval is $0.01-0.43$ by 0.06 ). The fraction of the tropics (i.e., $35^{\circ} \mathrm{S}-35^{\circ} \mathrm{N}$ ) covered by each regime is shown in the lower right of each panel. Crosses show the area-weighted mean and standard deviation for the regime. Black lines denote the APE SST and red lines are SST +2 simulations. The dashed line shows the empirical relation between stratus cover and stability from Klein and Hartmann (1993).

The Earth-like configurations of both models differ substantially from the aquaplanets in that the joint histograms of stability and cloud amount are skewed toward large cloud amount at high stability, especially within the subsidence regimes. We interpret this fea- ture as a signature of stratocumulus. As a guide, the empirical relation between low cloudiness and lowertropospheric stability from Klein and Hartmann (1993) is included in Fig. 9. The NCAR CAM distributions closely follow this relation. The cloud fraction param- 
eterization explicitly includes this relation, though the low-cloud amount is more strongly controlled by the parameterization's relative humidity dependence. However, the imposition of this empirical relation in the NCAR CAM might partly explain the tendency to produce more low cloud with increasing lowertropospheric stability within each dynamical regime. The GFDL AM simulations also capture the empirical relation, but with much more scatter.

The lack of a stratocumulus tail in the joint histograms of the aquaplanet simulations suggests that, to the extent aquaplanets reproduce the climate sensitivity of more realistic planets, differences among GCMs reflect differences in the treatment of shallow cumulus convection rather than stratocumulus. These results also support the association of stratocumulus with the zonally asymmetric component of circulation, which is missing in the aquaplanets, and trade cumulus with the zonally symmetric component of the circulation, which the aquaplanets well represent. That the aquaplanets reproduce the climate sensitivity of the standard configurations suggests that stratocumulus are not crucial to the climate sensitivity of either aquaplanets or more realistically configured models. While it could be argued that the absence of stratocumulus in aquaplanets in this analysis is an artifact of using monthly means, which tend to obscure transient and transitional features, repeating the analysis with daily averaged fields (not shown) produces similar results.

A remarkable aspect of these histograms is that the statistical mode of low-cloud amount varies neither among dynamic regimes nor with lower-tropospheric stability, especially for the aquaplanets. This suggests that vertical motion acts principally to sort clouds according to cloud-top temperature (or pressure), rather than isolating particular kinds of clouds. That is, low clouds are likely to cover about $30 \%$ of the area, independent of the large-scale environment, while deeper clouds are more prevalent in regions of ascent and inhibited in subsidence regimes. This independence of low-cloud amount from large-scale vertical motion runs counter to expectations, which would anticipate the mode of low cloudiness to increase with stronger descent (cf. Slingo 1980). Once again, the failure of such a result to emerge from the simulations does not appear to be an artifact of the analysis method. Using daily means, rather than monthly means, shows somewhat more variability in the mode of low-cloud amount among regimes, but a trend toward more low-cloud amount with increasing subsidence is present neither in the mode nor the mean.

Construction of such histograms is guided by, and complements, the dynamical regimes perspective of
Fig. 8. As mentioned, the dynamic term of Eq. (2) is associated with changes in the pdf of $\omega_{500}$, and the expression of that change is the change in area of largescale regimes. The thermodynamic effect arises from changes in the cloud properties, which are closely related to the cloud amount here, though conceivably it could be related to either optical properties or cloud morphology as well. Regimes in which the two terms are the same sign have large effects on the system. Weak subsidence regimes in most NCAR CAM cases, for example, dominate the overall cloud response because the tropical area covered by this regime increases (dynamic), and larger mean low-cloud amount within that area also increases (thermodynamic), in the SST + 2 simulations. On the other hand, the same weak subsidence regime in the GFDL AM has a decrease in mean low-cloud amount, but stronger (negative) CRF (opposing the overall effect; see Fig. 4), driven primarily by the dynamic influence of the regime covering more of the tropics in the SST +2 simulations. In the strong subsidence regimes, the NCAR CAM has an increase in mean low-cloud amount, but a decrease in the faction of the tropics with such strong subsiding motion, while the GFDL AM experiences a loss of lowcloud amount and a loss of area, leading to a weaker shortwave cloud forcing.

Large dynamic contributions in different circulation regimes could point to differences in regional circulation response as a source of disagreement in GCMs. However, by comparing the fraction of the tropics covered by these regimes, for example, as reported in Fig. 9 , it seems that by and large the GCMs agree on the areal coverage of the regimes as well as the change in area in the SST +2 cases. Thus, the source of disagreement among the models still seems to be the low-cloud effects, especially in subsidence regimes, and is likely to arise at least partially from differences in the representation of shallow cumulus convection, the dominant cloud type in subsidence regions in tropical and subtropical latitudes. The similarity in the distribution of vertical motion and cloud radiative response between aquaplanets and the more Earth-like version of the same GCM combined with the absence of subtropical stratocumulus in the aquaplanets further focuses attention on shallow cumulus convection as a key difference in the simulated climate response.

\section{Possible stratiform effects}

Concluding that shallow cumulus convection is a leading source of disagreement among the aquaplanet configurations is well supported above, but there remains a possibility that the standard configurations are influenced by changes in stratiform cloudiness. Figure 9 

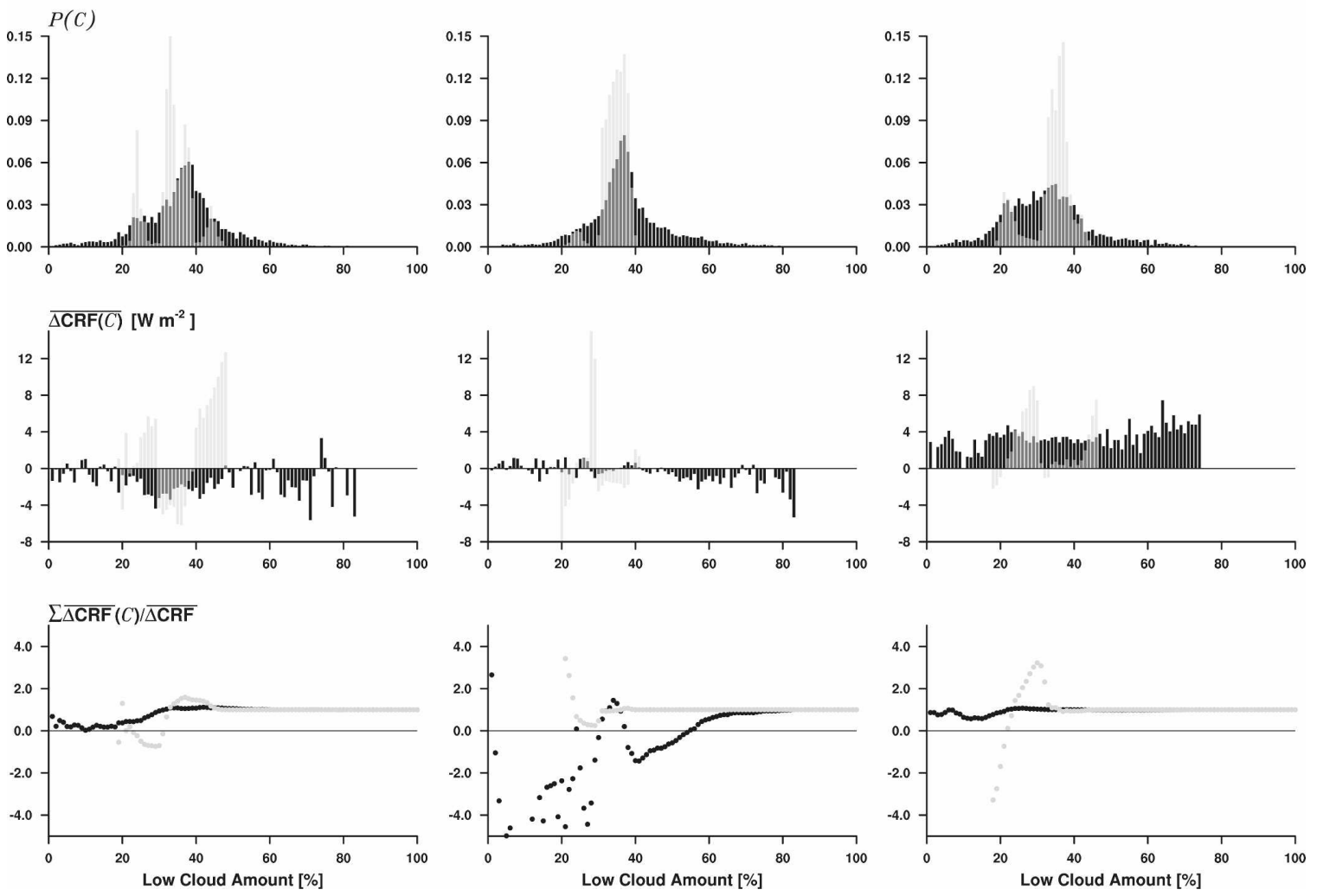

FIG. 10. (top) Distribution of low-cloud fraction in subsidence regimes $P(C)$ for the Earth-like configuration (black) and aquaplanet B (gray) for each GCM, as noted at the top of each column. Monthly mean output is used to generate the distribution for the control simulation, and the time series is averaged before constructing the distribution. (middle) The average change in CRF within each $1 \%$ bin of low cloud fraction from Eq. (4). (bottom) The cumulative change in CRF [Eq. (6)] as a fraction of the total change.

suggests that regions of persistent stratiform cloudiness are rare, but if the changes in stratiform cloudiness are sufficiently large, it might compensate for the rarity of such conditions. On shorter time scales, periods of stratiform cloudiness may also be critical to determining the monthly mean climatology. These possible roles of stratiform cloud types in the standard configuration simulations are considered briefly in this section.

Focusing on tropical subsidence regimes $\left(\omega_{500}>0\right)$, the distribution of low-cloud amount can be constructed for each of the GCMs. Figure 10 shows such distributions for the GFDL AM and both resolutions of the NCAR CAM for the standard (black) and aquaplanet B (gray) configurations (top panels). The distributions show the probability of low-cloud fraction $C$ in $1 \%$ bins $P(C)$. Conditions with prevailing cloudiness, for example, $C>50 \%$, cover a small area of the tropical belt for both the aquaplanet and Earth-like configurations. Aquaplanets have much narrower distributions, but share a mode (albeit more pronounced) with the Earth-like configurations. The peak of the distribution carries the largest statistical weight for changes in
$\mathrm{CRF}$, and changes in the predominant cloud regimes largely determine the overall change in CRF. This point is demonstrated by the middle panels of the figure, which show the average change in CRF conditioned on low-cloud fraction in the control simulation, described by

$$
\overline{\Delta \mathrm{CRF}(C)}=\frac{\sum_{i=1}^{N_{C}} \Delta \mathrm{CRF}_{i} a_{i}}{\sum_{i=1}^{N_{C}} a_{i}},
$$

where $a$ is the grid-cell area and $N_{C}$ is the number of grid points within the discrete interval associated with cloud fraction $C$; note that each value is weighted by the area with cloud fraction $C$, not the total tropical area. The average change in CRF over the entire sample (e.g., tropical subsidence locations) is given by weighting each $1 \%$ bin by its probability

$$
\overline{\Delta \mathrm{CRF}}=\sum_{i=0}^{I} \overline{\Delta \mathrm{CRF}\left(C_{i}\right)} P\left(C_{i}\right)
$$


where $I$ denotes the total number of intervals covering the full range of $C$; that is, summing the product of the distributions in the top and middle panels of Fig. 10. The relation between the most common cloud fraction and the overall change in CRF is most apparent for the aquaplanets, where the response of the predominant cloud regime mirrors the tropically averaged response. Large changes in CRF are often associated with relatively uncommon cloud fraction (both large and small), but the rarity of these clouds makes their contribution to the overall response modest; conversely, small changes in CRF over large areas heavily influence the overall change in CRF, as is most clear for the GFDL AM aquaplanet B.

The standard, Earth-like configurations are also most strongly influenced by the prevailing cloud types. One measure of the influence of different cloud fractions on the overall response is shown by the cumulative change in CRF, given by

$$
\overline{\Delta \mathrm{CRF}}(C)=\sum_{C_{i} \leq C} \overline{\operatorname{\Delta CRF}\left(C_{i}\right)} P\left(C_{i}\right) .
$$

The bottom panels of Fig. 10 show the ratio of this cumulative effect and the total response, $\overline{\Delta C R M}$. The cumulative change in CRF suggests that the cloudiest areas weakly contribute to the overall change; the influence of these clouds is offset by their rarity. At T42, the NCAR CAM shows a strengthening of (negative) CRF across the peak of the low-cloud fraction distribution, with almost no influence from large-cloud fractions. The higher-resolution NCAR CAM has a broader distribution of low cloud, hinting at a greater role for more stratiform cloud regimes. But even in this case cloud regimes for which the cloud fraction is greater than about $60 \%$ play little role, despite the tendency for the change in the CRF to increase in magnitude as cloudiness becomes more stratiform. The GFDL AM standard configuration also shows a broad distribution of low-cloud amount, though shifted to lower-cloud fraction relative to the NCAR CAM. It evinces a more coherent change in CRF across values of low-cloud fraction, meaning that the statistical weight, that is, the area covered, determines the influence of the cloud cover on the climate sensitivity, and most of that weight lies in cloud regimes corresponding to cloud fractions between $20 \%$ and $40 \%$. Using the complementary cumulative change in $\mathrm{CRF}$, that is, summing from $100 \%$ to $0 \%$ in Eq. (6), also suggests that the large statistical weight of cloud fractions less than $50 \%$ heavily influences the overall response. Based on these results, we believe that the bulk of the response in all these experiments is carried by the trade wind cumulus regions, though the tendency for strati- form cloudiness to play more of a role in the Earth-like simulation in the T85 case leaves room for some skepticism on this point.

The second possible effect of stratiform clouds on the climate sensitivity of these models is through transient cloudy conditions that monthly mean output might obscure. To better understand both the areal and vertical distribution of the simulated cloud fields, daily means are used to construct histograms of cloud fraction at each model level; an example is shown in Fig. 11 for the NCAR CAM at T85. These distributions show little evidence that periods of stratiform clouds are essential to the time-averaged response of the distribution. At most model levels, the daily mean cloud fraction is very small, while even the cloudiest levels rarely reach cloud fractions of more than about $30 \%$. The lowest levels of the NCAR CAM have about a 5\% occurrence of cloud fraction greater than $50 \%$, commensurate with the total low-cloud amount in the monthly distributions, which show about $10 \%$ chance of such cloud cover (Fig. 10). At any given model level, the mean (or mode) cloud fraction is less than the mean low-cloud fraction shown above; this is because of vertical integration combined with cloud overlap assumptions. The aquaplanet and standard configurations have similar cloud cover, in terms of both the vertical structure and the distribution of cloud cover. The equivalent distributions for the warmer SST simulations are very similar to the control simulations; the difference shows no preference for changes at large or small cloud fraction. Figure 11 shows only the NCAR CAM at T85, but the same features are evident at the lower resolution; daily output from the GFDL AM for these experiments is more limited, but also shows little evidence for periods of overcast conditions playing a large role in determining the character of the monthly averaged statistics. Similar analysis using condensate instead of cloud fraction (not shown) produces similar characteristics for all configurations.

This investigation of the role of large cloud fraction regions in the standard GCM configurations shows relatively little evidence that the stratiform response is important to the overall tropical change in CRF. Trade wind cumulus conditions prevail across a large portion of the tropics, producing modest low-cloud fraction. The large statistical weight of these areas ensures that they are important for the tropically averaged cloud response. Conversely, the small statistical weight in the tail of the low-cloud distribution, and the generally modest change in $\mathrm{CRF}$ in these regions, marginalizes the overall influence of the stratiform response. There is also no evidence for transient stratiform conditions 


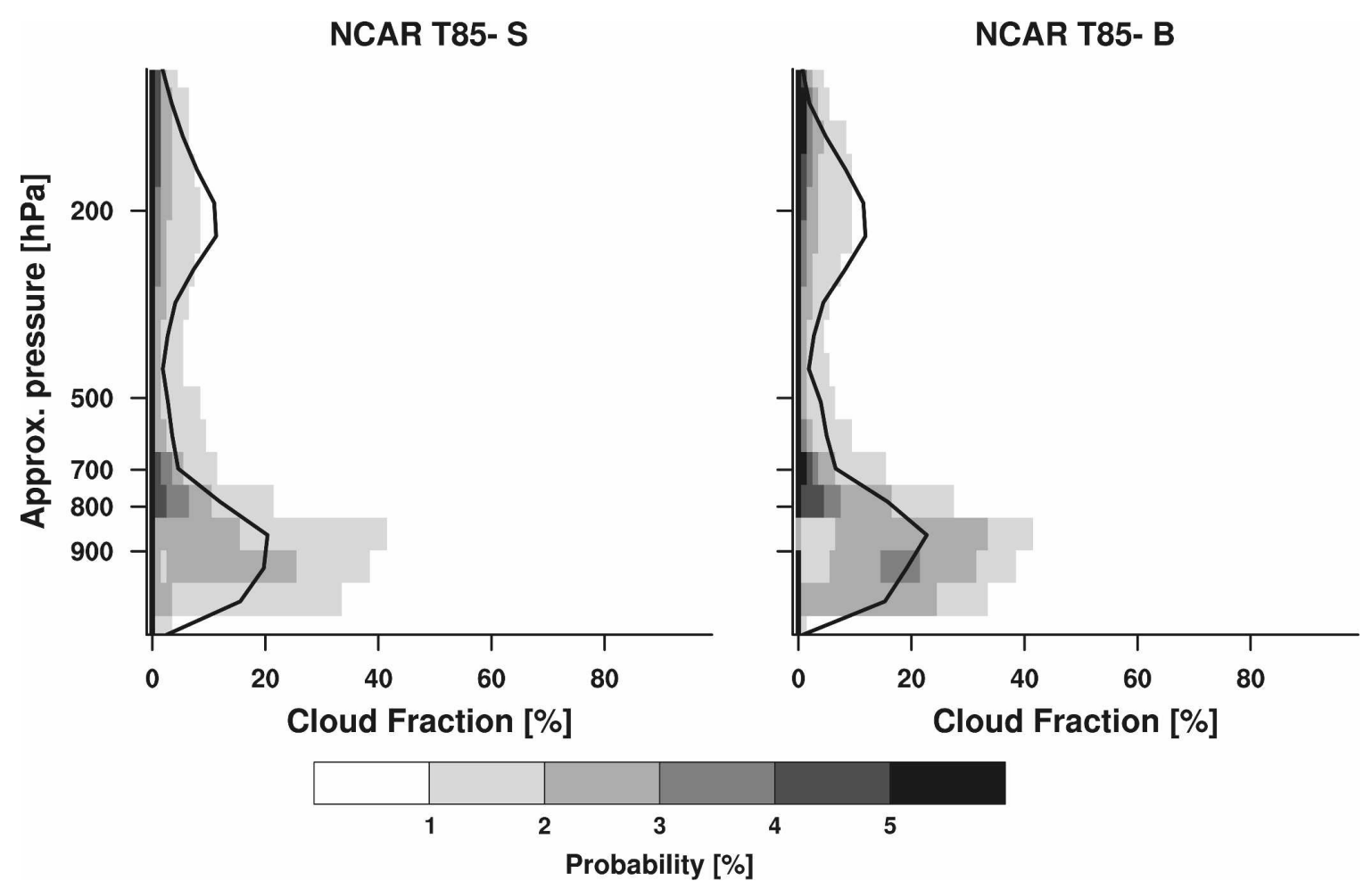

FIG. 11. Histogram of daily mean cloud fraction for subsidence points $\left(\omega_{500}>0\right)$ at each model level for the NCAR CAM at T85 for the (left) standard, Earth-like configuration and (right) aquaplanet B. The histogram is the shaded distribution, showing the probability of cloud fraction. The solid black curve shows the mean cloud profile, also conditioned on $\omega_{500}>0$. The levels are given by the approximate pressure of the model level; no vertical interpolation has been performed.

strongly affecting the distribution of low-level cloud cover. The difference in climate sensitivity between the GCMs appears to arise largely from the response of the broad regions of moderate cloud coverage. Describing the nature of the cloud response in these regions could thus improve understanding of the difference between the models; such a detailed analysis of the cloud response is a topic of current efforts.

\section{Summary}

We have shown that aquaplanets successfully predict the sensitivity of tropical top-of-atmosphere radiative fluxes. The climate sensitivity is related to changes in cloud radiative forcing, which is, in turn, related to changes in the low-level cloud field. Two independent GCMs with very different low-cloud responses to the same prescribed climate change both produce aquaplanets that essentially mimic the standard configuration's climate response. By using several aquaplanets for each GCM and comparing to the more Earth-like configuration, these cloud effects appear to be robust to moderate changes in large-scale circulations and derived from similar physical processes, suggesting that the representation of cloud processes are the ultimate, rather than a proximate, cause of such effects.

Sorting the simulation output by dynamic state corroborates the link between low clouds and climate sensitivity, and further shows that both the large-scale circulation and parameterized physics play a role. Aggregating dynamic states, dynamical regimes are constructed (Fig. 8) which show that weakly subsiding regions contribute most to the stronger (negative) CRF for the NCAR CAM, while strongly subsiding regimes are most important for the weakening CRF in the GFDL AM. Subsidence regimes are related to lowcloud types, such as stratocumulus and shallow cumulus, reinforcing the idea that low clouds are controlling climate sensitivity in the tropics. An investigation of the joint distributions of lower-tropospheric stability and low-cloud amount (Fig. 9) extends the dimensionality of the dynamical regimes analysis and sheds light on the relationship of cloud response and thermodynamic state. The distributions indicate a dearth of stratocumulus in the aquaplanets, which, combined with their similar climate sensitivity and overall cloud response to the more Earth-like simulations, suggests that the representation of shallow (trade wind) cumulus convection 
in these climate models underlies their differing climate sensitivity.

To test whether stratocumulus might be playing an important role in the response of the Earth-like configurations, the distribution of cloud in space and time is investigated. Large values of low-cloud fraction are shown to cover small areas of the tropics, giving little statistical weight to the cloud response in those regions. That response can be sizeable, but generally is overwhelmed by the much larger area covered by more modest cloud fraction. Daily mean cloud fraction is also used to demonstrate that brief periods of large-cloud fraction are not determining the radiative properties of the low-cloud regimes. That is, transient low-level cloud appears to play little role in determining either the cloud fraction or the change in cloud fraction in these experiments. These results provide confidence in the inference that shallow cumulus clouds are crucial to the disagreement in climate sensitivity of these GCMs, and that the aquaplanet experiments correctly capture this disagreement.

One limitation of the simulations is their lack of a closed surface energy budget. An approach that could maintain the elegance of the current framework, yet address this issue, would be to compare aquaplanets with a mixed layer ocean and idealized profiles of meridional heat transport for present-day and doubled $\mathrm{CO}_{2}$ concentrations. Another limitation of the current work is its lack of stratocumulus in the aquaplanets, a climatological cloud type thought to be fundamental to the current-day radiative balance, even if they do not appear to play an important role in explaining the differences in the climate sensitivity of the two models explored here. In situations where distinctly stratocumulus effects are desired, the zonally symmetric aquaplanet will be inadequate. In such cases, introducing an idealized zonal asymmetry, for example, a wavenumber 2 SST pattern or oceanic meridional heat transport in the subtropical latitudes, might allow one to use the aquaplanet framework for exploring the effects associated with stratocumulus.

Based on the results presented here, several avenues for future investigation seem promising. Comparing additional GCMs in similar experiments as described here could provide further insight into the disagreement among current-generation GCMs in terms of cloud effects. Sensitivity studies using aquaplanet conditions within a single GCM could shed light on the origin of cloud effects in that particular model, especially through systematic changes to the cloud parameterization within a single dynamical framework. Aquaplanet simulations can also provide rich forcing datasets for process studies with high-resolution models, such as single-column models or large-eddy simulation, as well as conditionally averaged statistics that can readily be compared to data. These suggestions broadly follow previous discussion of aquaplanets (Hoskins et al. 1999), but extend the framework to investigate climate sensitivity, with an emphasis on better understanding cloud effects and improving the representation of clouds in large-scale models.

Acknowledgments. We would like to thank the editors and reviewers for helpful comments. This work has been supported by NOAA Grant NA17RJ1232AM021 and NSF Grant ATM-0336703, and by the NSF Science and Technology Center for Multi-Scale Modeling of Atmospheric Processes, managed by Colorado State University under Agreement ATM-0425247.

\section{REFERENCES}

Arakawa, A., 1975: Modelling clouds and cloud processes for use in climate models. The Physical Basis of Climate and Climate Modelling, GARP Publication Series, Vol. 16, ICSU/WMO, 181-197.

Bony, S., and J.-L. Dufresne, 2005: Marine boundary layer clouds at the heart of tropical cloud feedback uncertainties in climate models. Geophys. Res. Lett., 32, L20806, doi:10.1029/ 2005GL023851.

,-- , H. L. Treut, J.-J. Morcrette, and C. Senior, 2004: On dynamic and thermodynamic components of cloud changes. Climate Dyn., 22, 71-86.

Cess, R., and Coauthors, 1989: Interpretation of cloud-climate feedback as produced by 14 atmospheric general circulation models. Science, 245, 513-516.

- and Coauthors, 1990: Intercomparison and interpretation of climate feedback processes in 19 atmospheric general circulation models. J. Geophys. Res., 95, 16 601-16 615.

- , and Coauthors, 1996: Cloud feedback in atmospheric general circulation models: An update. J. Geophys. Res., 101, $12791-12794$.

Chao, W. C., and B. Chen, 2004: Single and double ITCZ in an aqua-planet model with constant sea surface temperature and solar angle. Climate Dyn., 22, 447-459.

Charney, J. G., and Coauthors, 1979: Carbon Dioxide and Climate: A Scientific Assessment. National Research Council, 22 pp.

Collins, W. D., and Coauthors, 2006: The Community Climate System Model version 3 (CCSM3). J. Climate, 19, 2122-2143.

Delworth, T. L., and Coauthors, 2006: GFDL's CM2 global coupled climate models. Part I: Formulation and simulation characteristics. J. Climate, 19, 643-674.

Hack, J. J., 1994: Parameterization of moist convection in the National Center for Atmospheric Research community climate model (CCM2). J. Geophys. Res., 99, 5541-5568.

Hayashi, Y.-Y., and A. Sumi, 1986: The 30-40 day oscillations simulated in an "aqua planet" model. J. Meteor. Soc. Japan, 64, 451-467.

Held, I. M., and B. J. Soden, 2000: Water vapor feedback and global warming. Annu. Rev. Energy Environ., 25, 441-475. , and - 2006: Robust responses of the hydrological cycle to global warming. J. Climate, 19, 5686-5699. 
Holtslag, A. A. M., and B. A. Boville, 1993: Local versus nonlocal boundary-layer diffusion in a global climate model. J. Climate, 6, 1825-1842.

Hoskins, B., R. Neale, M. Rodwell, and G.-Y. Yang, 1999: Aspects of the large-scale tropical atmospheric circulation. Tellus, 51A, 33-44.

Houghton, J., Y. Ding, D. Griggs, M. Noguer, P. van der Linden, X. Dai, K. Maskell, and C. Johnson, Eds., 2001: Climate Change 2001: The Scientific Basis. Cambridge University Press, $944 \mathrm{pp}$

Kiehl, J. T., 1994: On the observed near cancellation between longwave and shortwave cloud forcing in tropical regions. $J$. Climate, 7, 559-565.

Kirtman, B. P., and E. K. Schneider, 2000: A spontaneously generated tropical atmospheric general circulation. J. Atmos. Sci., 57, 2080-2093.

Klein, S. A., and D. L. Hartmann, 1993: The seasonal cycle of low stratiform clouds. J. Climate, 6, 1587-1606.

Larson, K., D. L. Hartmann, and S. A. Klein, 1999: The role of clouds, water vapor, circulation, and boundary layer structure in the sensitivity of the tropical climate. J. Climate, 12, 23592374.

Lock, A. P., A. R. Brown, M. R. Bush, G. M. Martin, and R. N. B. Smith, 2000: A new boundary layer mixing scheme. Part I: Scheme description and single-column model tests. Mon. Wea. Rev., 128, 3187-3199.

Miller, R. L., 1997: Tropical thermostats and low cloud cover. $J$. Climate, 10, 409-440.

Miura, H., H. Tomita, T. Nasuno, S.-I. Iga, M. Satoh, and T. Matsuno, 2005: A climate sensitivity test using a global cloud resolving model under an aqua planet condition. Geophys. Res. Lett., 32, L19717, doi:10.1029/2005GL023672.

Moorthi, S., and M. J. Suarez, 1992: Relaxed Arakawa-Schubert. A parameterization of moist convection for general circulation models. Mon. Wea. Rev., 120, 978-1002.
Neale, R., and B. Hoskins, 2001: A standard test for AGCMs including their physical parameterizations: I: The proposal. Atmos. Sci. Lett., 1, 101-107.

Randall, D., and Coauthors, 2007: Climate models and their evaluation. Climate Change 2007: The Physical Science Basis, S. Solomon et al., Eds., Cambridge University Press, 589-682.

Ringer, M. A., and Coauthors, 2006: Global mean cloud feedbacks in idealized climate change experiments. Geophys. Res. Lett., 33, L07718, doi:10.1029/2005GL025370.

Slingo, J. M., 1980: A cloud parameterization scheme derived from GATE data for use with a numerical model. Quart. J. Roy. Meteor. Soc., 106, 747-770.

Soden, B. J., A. J. Broccoli, and R. S. Hemler, 2004: On the use of cloud forcing to estimate cloud feedback. J. Climate, 17, 3661-3665.

— coupled ocean-atmosphere models. J. Climate, 19, 3354 3360 .

Tiedtke, M., 1993: Representation of clouds in large-scale models. Mon. Wea. Rev., 121, 3040-3061.

Williamson, D. L., and J. G. Olson, 2003: Dependence of aquaplanet simulations on time step. Quart. J. Roy. Meteor. Soc., 129, 2049-2064.

Wood, R., and C. S. Bretherton, 2006: On the relationship between stratiform low cloud cover and lower-tropospheric stability. J. Climate, 19, 6425-6432.

Wyant, M. C., C. S. Bretherton, J. T. Bacmeister, J. T. Kiehl, I. M. Held, M. Zhao, S. A. Klein, and B. J. Soden, 2006: A comparison of tropical cloud properties and responses in GCMs using mid-tropospheric vertical velocity. Climate Dyn., 27, 261-279, doi:10.1007/s00382-006-0138-4.

Zhang, G. J., and N. A. MacFarlane, 1995: Sensitivity of climate simulations to the parameterizations of cumulus convection in the Canadian Climate Centre general circulation model. Atmos.-Ocean, 33, 407-446. 